\title{
GOÄ-Abrechnung
}

\section{Höheren Gebührensatz richtig begründen}

\author{
Schwierigkeiten bei der Leistungserbringung, besondere Umstände \\ und erhöhter Zeitaufwand sind mögliche Begründungen für einen \\ erhöhten Faktor in der Privatabrechnung. Doch um Ärger zu \\ vermeiden, ist es wichtig, sich möglichst wenig angreifbar zu machen.
}

D iese Flexibilität haben Sie nur bei Privatpatienten: Sie können einen Faktor zwischen dem einfachen und dem dreieinhalbfachen des Gebührensatzes wählen. Ob das nach einer GOÄ-Novelle so bleibt, ist derzeit ungewiss, aber bis dahin sind Sie frei bei der Wahl des Faktors - je nach Aufwand, der Ihnen bei der Betreuung des Patienten entsteht. Für die Steigerung zwischen dem 2,31-fachen und dem 3,5-fachen Satz müssen Sie allerdings eine Begründung beifügen, die den höheren Aufwand rechtfertigt. Achten Sie dabei darauf, dass Sie sich nicht angreifbar machen. So sorgen Sie dafür, dass Ihre Patienten keinen Ärger mit der Versicherung bekommen - und Sie nicht in der Folge mit dem Patienten.

Die in der GOÄ geforderte Begründung beim Überschreiten des 2,3-Fachen des Gebührensatzes bereitet vielen Ärzten Probleme, weil Patienten und

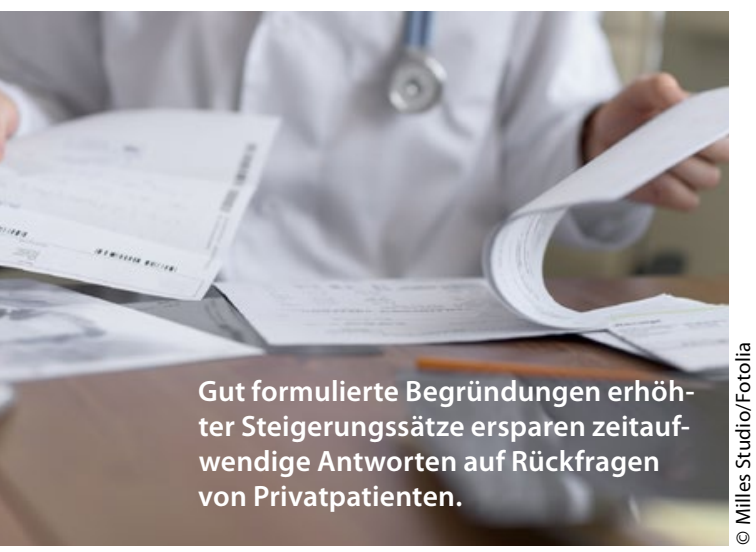

Privatversicherungen zunehmend die Steigerungspraxis auf einen höheren als den 2,3-fachen Gebührensatz monieren, wenn keine Begründung gegeben wird.

Nicht selten liegt es aber auch an der Art der Begründung. Ein Blick in Paragraf 5 GOÄ bringt etwas Klärung: „Innerhalb des Gebührenrahmens sind die Gebühren unter Berücksichtigung der Schwierigkeit und des Zeitaufwandes der einzelnen Leistungen sowie der Umstände bei der Ausführung nach billigem Ermessen zu bestimmen. Die Schwierigkeit der einzelnen Leistung kann auch durch die Schwierigkeit des Krankheitsfalles begründet sein. (...) In der Regel darf eine Gebühr nur zwischen dem Einfachen und dem 2,3-fachen des Gebührensatzes bemessen werden; ein Überschreiten des 2,3-fachen des Gebührensatzes ist nur zulässig, wenn Besonderheiten der in Satz 1 genannten Bemessungskriterien dies rechtfertigen."

\section{Zeitaufwand ist oft umstritten}

Es sind also eindeutig die Besonderheiten aufgeführt, die eine Faktorerhöhung über den 2,3-fachen Satz (bei persönlichen Arztleistungen) begründen beziehungsweise erklären: Schwierigkeit, Umstände (der Leistungserbringung) und Zeitaufwand, sind zentrale Kriterien. Die Begründung „erhöhter Zeitaufwand“ löst auch bei Privatpatienten immer einen gewissen Zweifel aus und bietet somit Anlass zu Diskussionen. Ebenso wie der GKV-Patient hat auch der Privatpatient fast immer das Gefühl, dass er viel zu kurz beim Arzt persönlich war und die Behandlung doch gar nicht so lange gedauert hat. Aus diesem Grund sollten Sie die Begründung „erhöhter Zeitaufwand" aus Ihrem Repertoire streichen. Zumindest sollten Sie deren Verwendung immer kritisch überdenken.

Bei den Umständen ist diplomatisches Vorgehen gefragt: Ist es vielleicht ein unruhiger Patient, ein älterer Patient mit demenzieller Entwicklung, der bestimmte Aufforderungen zur diagnostischen Leistungsdurchführung nicht versteht und entsprechend umsetzt? Dann sollten Sie das in der Begründung auch differenziert und nicht nur pauschal begründen.

Auch bei der Schwierigkeit des Krankheitsbildes ist Vorsicht geboten. Mit der Begründung, die Erkrankung sei schwierig, schwerwiegend oder kompliziert, verursachen Sie letztlich Angst beim Patienten. Es können Rückfragen entstehen wie: „Herr Doktor, bin ich wirklich so krank?"

\section{Weichere Begründungen sind hilfreich}

Mit solchen Begründungen machen Sie sich angreifbar. Deshalb sollten Sie „weichere" Begründungen formulieren. Hier einige Beispiele von Begründungen, die kaum Anlass zu Rückfragen geben, weder von der Versicherung, noch seitens des Patienten:

Erhöhter Zeitaufwand: „Beratungsintensives Krankheitsbild" wählen, oder aber „differenzialdiagnostischer Aufwand bei komplexem Krankheitsbild“.

Umstände oder Schwierigkeitsgrad: „Beratung bei komplexem differenzialdiagnostischen Krankheitsbild" bietet ebenso wenig Angriffsfläche wie „komplexer differenzialtherapeutischer Ansatz".

Peter Schlüter 